\title{
Sham surgery: justified but practical? A systematic review of sham surgery trials in orthopaedics
}

\author{
Bunzli $\mathrm{S}^{1^{*}}$, Dowsey $\mathrm{MM}^{2^{*}}$, Choong $\mathrm{PF}^{3^{*}}$ \\ ${ }^{1}$ BPhty, PhD; Research Associate \\ 2 BHealthSci, MEpi, PhD; Associate Professor, Senior Principle Research Fellow \\ ${ }^{3}$ MBBS, MD, FRACS, FAOrthA, FAAHMS); Professor of Surgery, Head of Department of Surgery and Director of Orthopaedics, St Vincent's Hospital \\ *The University of Melbourne, Department of Surgery, St Vincent's Hospital, Victoria, Australia
}

Corresponding author: Prof Peter Choong, The University of Melbourne, Department of Surgery, St Vincent's Hospital, Level 2, Clinical Sciences Building, 29 Regent Street, Fitzroy 3065, Victoria, Australia; email: pchoong@unimelb.edu.au; tel: +61 392313955

\begin{abstract}
Background: An increasing trend for sham surgery trials in minor orthopaedic procedures has been observed. Trial outcomes have changed the practice landscape of these procedures. However, there has been no sham surgery trial in a major orthopaedic procedure. The aims of this systematic review were to consider the ethics of sham surgery trials; to describe orthopaedic sham surgery trials conducted to date; and to consider the challenges that will need to be overcome in order to conduct sham surgery trials for major orthopaedic procedures in the future.

Methods: A systematic review of the literature and clinical trial registries was undertaken. Trials with a published main findings paper underwent a risk of bias assessment using the Cochrane Collaboration risk of bias tool, in addition to an ethical assessment based on the work of Horng and Miller.

Results: We identified 22 sham surgery trials for minor orthopaedic procedures that have been completed, terminated, or are currently in process. Among the ten trials with a published main findings paper, only one was free from risk of bias; all others were at risk of bias. According to the ethical assessment, the benefits of a sham control were outweighed by the risks in all but two of the ten trials. Across the 22 trials with published and unpublished main findings, participant recruitment within reasonable timeframes, as well as the low threshold for crossover from the sham were recurring challenges.

Conclusions: Researchers are obliged to carefully consider the feasibility of conducting a sham surgery trial in a major orthopaedic procedure, before drawing on limited research funds. Exploring the conditions under which patients and surgeons would find participation in a sham surgery trial acceptable, and simulating trial costs based on patient and surgeon preferences may assist funders, assessors and ethics boards to determine whether to support the conducting of future sham surgery trials in major orthopaedic procedures.
\end{abstract}

Level of evidence: Level 1

Key words: orthopaedics, sham surgery, placebo, systematic review

Citation: Bunzli S, Dowsey MM, Choong PF. Sham surgery: justified but practical? A systematic review of sham surgery trials in orthopaedics. SA Orthop J 2018;17(3):15-24. http://dx.doi.org/10.17159/2309-8309/2018/v17n3a1

Editor: Prof LC Marais, University of KwaZulu-Natal, Durban

Received: July $2018 \quad$ Accepted: July $2018 \quad$ Published: August 2018

Copyright: @ 2018 The authors. This is an open-access article distributed under the terms of the Creative Commons Attribution Licence, which permits unrestricted use, distribution and reproduction in any medium, provided the original author and source are credited.

Funding: Michelle Dowsey is supported by a Career Development Fellowship from the National Health and Medical Research Council of Australia. Peter Choong and Michelle Dowsey hold a National Health and Medical Research Council Centre for Research Excellence Grant in Total Joint Replacement (1116325). The sponsors had no role in the design, collection, data analysis or interpretation nor in the preparation of this manuscript. 


\section{Introduction}

The cornerstone of modern clinical practice, evidence-based medicine, is 'the conscientious, explicit and judicious use of current best evidence in making decisions about the care of individual patients.' ${ }^{1}$ Adequately powered, low risk of bias, randomised controlled trials (RCTs) sit at the top of the evidence hierarchy, providing the least biased evidence for the efficacy and safety of an intervention. Randomised allocation means prognostic factors are equally distributed between groups, reducing the influence of confounding factors on treatment outcome. Evidence-based decision-making in orthopaedic surgery is impeded by a paucity of RCTs. The majority of orthopaedic surgeries are not based on RCT evidence. ${ }^{2}$ But can we justify the widespread use of these procedures without rigorous testing?

In typical orthopaedic RCTs, patients are randomised to Surgery A or Surgery B. If both procedures are found to be equally effective, it is rarely reported that Surgery A and Surgery B might be equally ineffective or even equally harmful. Instead of comparing the efficacy of two procedures, a more relevant question is whether these procedures are superior to a placebo, or sham surgery. ${ }^{3}$

Sham surgery (involving anaesthesia and skin incision) enables researchers to determine whether the supposed 'therapeutic' element of the procedure has a benefit beyond a placebo effect. Placebo effects are the non-specific effects that can influence treatment outcomes. These include the rituals involved in undergoing the procedure, setting in which it is performed, status of the treating clinician, personal investment in the outcome, and patients' outcome expectations. Placebo effects are larger in surgical compared to non-invasive interventions, ${ }^{4}$ particularly interventions with subjective outcomes such as pain and function. ${ }^{5}$ Subsequently, placebo effects must be considered when interpreting the results of orthopaedic procedures seeking to improve quality of life (as opposed to preserve life).

An increasing trend for sham surgery trials for minor orthopaedic procedures has been observed. ${ }^{6-12}$ These trials have changed the practice landscape of these procedures. However, there has been no sham surgery trial in a major orthopaedic procedure. As such it has been argued that the efficacy of some of the highest volume and complex medical interventions such as joint replacement surgery remains unknown. ${ }^{2}$

This article discusses the ethical considerations of sham surgery trials and describes the current landscape of sham surgery trials in orthopaedics. Through a systematic review of the literature and search of clinical trial registries, we identified all sham surgery trials conducted to date for minor orthopaedic procedures, and considered the challenges that will need to be overcome in order to conduct sham surgery trials for major orthopaedic procedures in the future.

\section{Is it ethical to conduct a sham surgery trial?}

Much ethical debate surrounds the conduct of sham surgery trials. Offering no therapeutic benefit while exposing patients to unnecessary risks associated with anaesthesia and skin incision, sham surgery presents a threat to the ethical principles of beneficence and non-maleficence. A survey of shoulder surgeons found that $62 \%$ were concerned with the element of deception involved in performing sham surgery, and $48 \%$ were apprehensive about risking patient-surgeon trust. ${ }^{13}$ However, patients are exposed to surgical risks every day for interventions that may have no benefit beyond a placebo effect. The risks of adverse effects associated with sham surgery are low, and in some cases, may be even smaller than the surgical arm. ${ }^{14}$ Benefits may even come with study participation in the form of additional monitoring, clinical visits and interviews, providing attention and validation to patients. ${ }^{15}$ While surgeons must deceive their patients during the follow-up period to preserve blinding, this is not necessarily unacceptable to patients. As long as they are fully informed, some patients appear willing to participate in sham surgery trials in order to 'contribute to science'. ${ }^{16}$ With millions of people potentially exposed to surgical risks and financial costs for a placebo effect that may not last, it may be unethical not to conduct sham surgery trials. What do we tell patients why a procedure that is unproven is being offered to them?

\section{When are sham trials indicated?}

The Declaration of Helsinki states that the benefits, risks, burdens and effectiveness of an intervention must be tested against those of the best-proven intervention(s). A placebo control may be acceptable if: a) no proven intervention exists; b) where, for compelling and scientifically sound methodological reasons the use of a placebo is necessary to determine the efficacy or safety of an intervention; c) patients who receive the placebo will not be subject to additional risks of serious or irreversible harm from not receiving the best proven intervention. ${ }^{17}$ The American Medical Association guidelines ${ }^{18}$ propose that a sham surgery trial may be indicated if six ethical principles are met (Table 1).

Table 1. Guidelines for sham surgery trials (based on Tenery et al. ${ }^{18}$ )

The appropriateness of a surgical sham control should be evaluated on the following:

1. Sham controls should only be used when no other trial design will yield the same data.

2. Careful attention is paid to the informed consent process when enrolling participants.

3. Sham controls are not justified when testing the effectiveness of a minor modification to an existing procedure.

4. Sham controls may be justified if it is known that the disease being studied is susceptible to a placebo effect.

5. The risks of the sham control operation should be relatively small.

6. The patient must be offered the opportunity to receive the standard treatment if it is found to be efficacious at the end of the study.

\section{What sham surgery trials have been conducted in orthopaedics?}

This question is addressed in the Methods section.

\section{Methods}

\section{Design}

We conducted a systematic review of the published literature to identify sham surgery trials in orthopaedics with a published main findings paper. To better understand the current landscape of sham surgery trials in orthopaedics, we also searched clinical trial registries for trials that are currently underway, have been recently completed or have been abandoned. This systematic review is reported according to PRISMA guidelines. ${ }^{19}$

\section{Identification and selection of studies}

Five electronic databases (Medline, Embase, PsychInfo, Cochrane Register of Clinical Trials, CINAHL) were searched from inception to June 2018. The search strategy comprised two key concepts: surgery and placebo/sham. For each concept, key words and MeSH 
(Medical Subject Heading) terms were combined using the 'OR' operator and the results were combined using the AND operator. An example of the search in one database can be viewed in Table II. We limited searches to English language, humans and RCTs. The search results were downloaded into a bibliographic software. Reference lists of selected articles were manually searched for additional relevant articles.

\section{Table II: Search strategy in Medline}

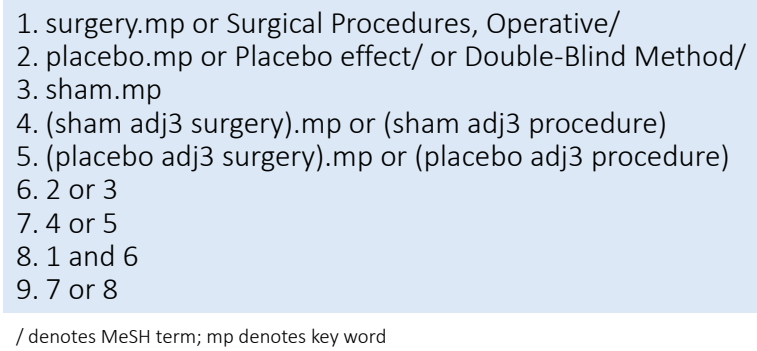

Two reviewers independently reviewed the titles and abstracts yielded according to the inclusion criteria. If eligibility was uncertain based on title and abstract, the full-text article was obtained. We included studies that randomised adults to receive either an invasive orthopaedic procedure or a sham procedure. We defined an invasive orthopaedic procedure as a surgical procedure addressing morphological structures of the musculoskeletal system. As such, we did not include studies of radiofrequency denervation or other nerve ablation procedures. We considered a procedure to be a sham when it mimicked the invasive procedure under investigation but did not change the integrity of the body part being operated. Therefore, we included studies that involved diagnostic arthroscopy as a sham but excluded studies that involved arthroscopic debridement as a sham. Results from the screening process by the two authors were compared and discrepancies were resolved through consensus discussion.

In addition to the database search, we searched the following clinical trial registries: Clinicaltrials.gov, Australian New Zealand Clinical Trials Registry, European Union Clinical Trials Registry, and Chinese Clinical Trial Registry using the key search terms: surgery and placebo/sham.

\section{Risk of bias assessment}

Two authors independently assessed each of the included studies with a published main findings paper using the Cochrane Collaboration's tool for assessing risk of bias in randomised trials. ${ }^{20}$ Assessments between the two authors were compared and discrepancies were resolved through consensus discussion with the third author. As this review aimed to identify the challenges facing sham surgery trials in orthopaedics, we did not exclude any studies on the basis of bias. Published protocol papers and unpublished studies identified from the clinical trials registries did not undergo a risk of bias assessment due to a lack of available information and their incomplete nature.

\section{Ethical assessment}

Each of the included studies with a published main findings paper also underwent an ethical assessment, based on the work of Horng and Miller. ${ }^{21}$ Comprising of six questions, the assessment estimates the risk-benefit ratio of the use of a sham control in surgery. ${ }^{22}$ Two authors independently performed the assessment. Findings were compared, and discrepancies were resolved through consensus discussion with the third author.

\section{Data extraction and analysis}

We extracted the following data from the studies retrieved (where provided): participant characteristics, proportion of eligible candidates that agreed to participate, sample size (actual and anticipated), time to recruit the sample, procedure(s) under investigation, trial status (e.g. completed, recruiting, abandoned), blinding procedures and success of blinding, study outcomes and adverse events. We compared the main findings papers to published protocol papers where available, and details registered in clinical trial websites to identify any modification to trial protocols. We summarised the trials in tabulated format and descriptive text. We presented the trials which had published a main findings paper separately to trials which had not.

\section{Findings}

The database searches identified 1306 articles for title and abstract screening. Of these, 22 were retrieved for full-text screening. Two articles were excluded as they involved a nonsurgical sham. Two articles were protocol papers of trials that were yet to publish a main findings paper. Eighteen articles (nine trials) met the inclusion criteria. One further trial was identified from handsearching references from the included studies, bringing the total sample of trials with a published main findings paper to ten. Registry searches identified a further 12 sham surgery trials. Four trials had been completed but were yet to publish a main findings paper, one had converted to a cohort study, and seven trials were currently underway. The yield of studies is presented in Figure 1.

The 22 sham surgery trials both with and without a main

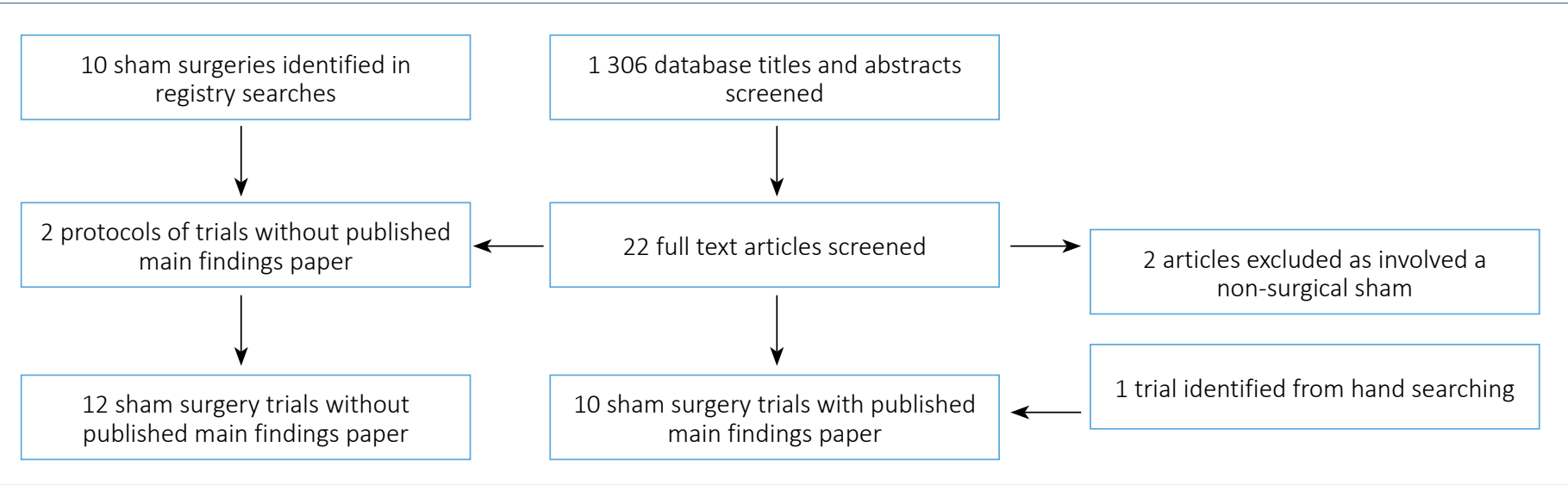

Figure 1. Yield of studies 


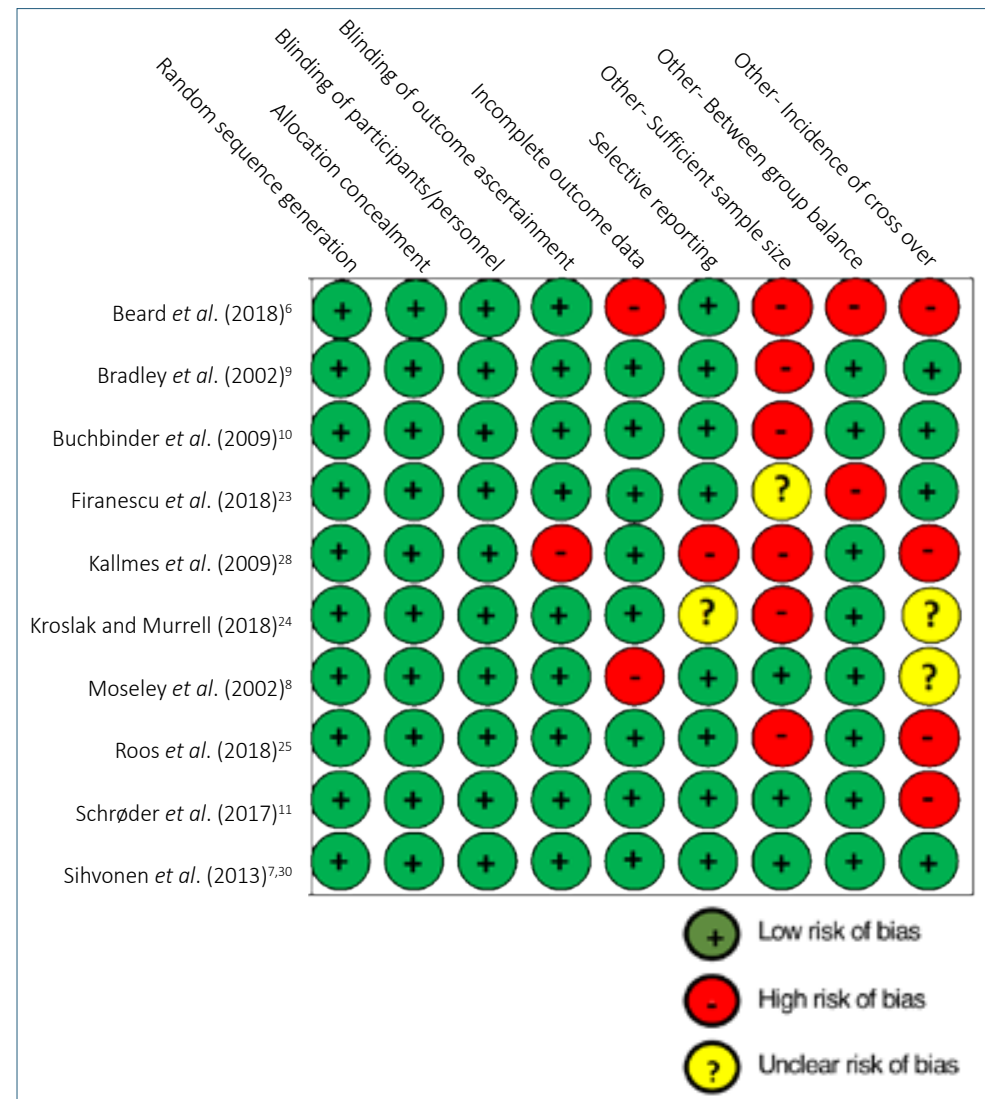

Figure 2. Risk of bias assessment

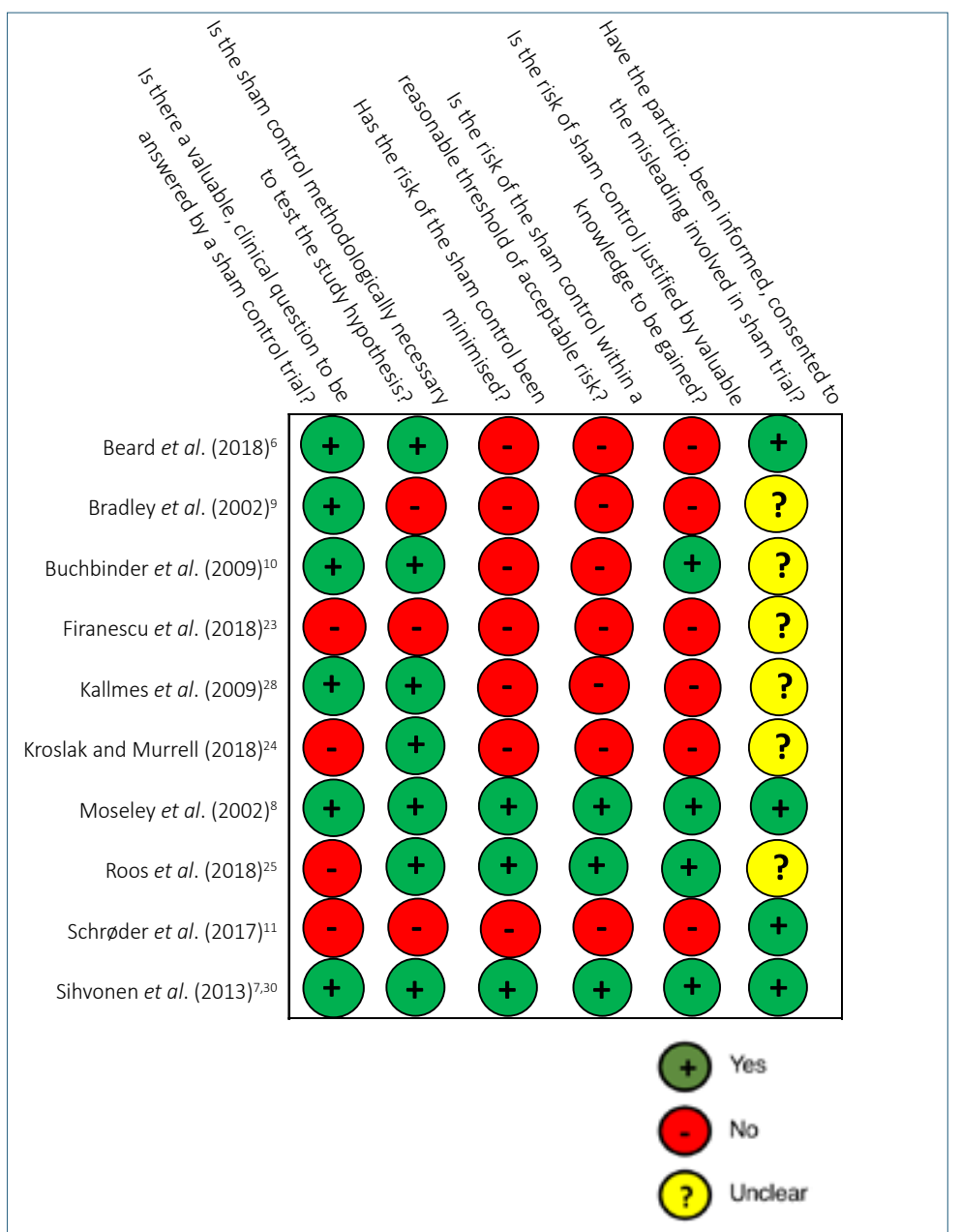

Figure 3. Ethical assessment findings paper, involved the following orthopaedic procedures: vertebroplasty $(n=3)$, decompressive spinal surgery $(n=3)$, stem cell procedures for knee osteoarthritis $(n=3)$, and arthroscopic procedures of the knee $(n=6)$, hip $(n=1)$, shoulder $(n=4)$, and elbow $(n=2)$.

\section{Findings from trials with a published main findings paper}

Risk of bias among the ten included trials with a main findings paper is presented in Figure 2. All studies described effective randomisation and allocation concealment. All described appropriate blinding procedures; however, not all assessed the success of blinding by asking participants if they believed they had undergone the intervention or sham.

Risk of bias was introduced in most trials due to revision of the target sample size during the course of the trial or failing to reach target sample size. We considered this source of bias to be different from incomplete outcome data as follow-up rates for participants that were enrolled were generally high. The Cochrane Collaboration risk of bias tool enables authors to describe 'other sources of bias' that are not covered by the six domains; therefore, we chose to include 'sufficient sample size' as an assessment criterion.

Another source of bias we identified was imbalance of characteristics between groups. For example, in the trial by Beard et $a .^{6}$ there was imbalance in the proportion of participants who had undergone their allocated intervention or sham at the time of assessment ( $23 \%$ versus $42 \%$ respectively). While the authors attempted a sub-analysis of only those who had undergone the procedure, this was not sufficiently powered to detect between group differences. We considered this source of bias to be different from selection bias or incomplete outcome data, and therefore chose to include 'between group balance' as an assessment criterion.

A final source of bias we identified was crossover between the study arms during the follow-up period, where the threshold for crossover was lower in the sham group than the intervention groups. While ineffective blinding procedures may explain crossover, other factors such as treatment failure may play a role and therefore we chose to include 'incidence of crossover' as an assessment criterion.

Findings from the ethical assessment is presented in Figure 3. Four trials did not provide satisfactory evidence of a valuable, clinically relevant question to be answered by a sham surgery trial. Two involved surgical procedures in populations where this was not indicated at the time the study was designed: vertebroplasty for acute vertebral compression fractures ${ }^{23}$ and surgical management of tennis elbow in patients with less than 12 months of pain. ${ }^{24}$ Without including a no-treatment arm in these trials, it cannot be ruled out that these procedures were performed on people who might have naturally recovered. In one trial investigating the surgical management of type II SLAP (superior labral tear from anterior to posterior) lesions, ${ }^{11}$ little evidence was available at the time the trial was designed about non-operative management of these lesions suggesting that a sham surgery trial was premature. In another trial investigating an arthroscopic procedure for the knee, ${ }^{25}$ the authors acknowledged that there was already a sham surgery trial underway for this procedure when they registered the trial.

According to Horng and Miller, ${ }^{26}$ sham surgery should involve no more than anaesthesia and a skin incision mimicking the procedure under investigation to keep within the risk threshold of other accepted research interventions that do not offer participants direct benefits such as muscle biopsy. Seven trials 
Table III. Sham surgery trials with published main findings

\begin{tabular}{|c|c|c|c|c|c|}
\hline Study group & Comparison & Outcome & $\begin{array}{l}\text { Proportion of } \\
\text { eligible candidates } \\
\text { that agreed to } \\
\text { participate }\end{array}$ & $\begin{array}{l}\text { Target sample } \\
\text { size met? }\end{array}$ & $\begin{array}{l}\text { Time to } \\
\text { recruit } \\
\text { sample }\end{array}$ \\
\hline $\begin{array}{l}\text { Beard et al. } \\
(2018)^{6}\end{array}$ & $\begin{array}{l}\text { Arthroscopic subacromial } \\
\text { decompression to sham surgery } \\
\text { \& no treatment for shoulder pain }\end{array}$ & $\begin{array}{l}\text { No differences between groups at } \\
1 \text { year; both showed improvement over } \\
\text { no treatment }\end{array}$ & $40 \%$ & $\begin{array}{c}\text { Yes } \\
n=313\end{array}$ & 3 years \\
\hline $\begin{array}{l}\text { Bradley et al. } \\
(2002)^{9}\end{array}$ & $\begin{array}{l}\text { Needle tidal irrigation to sham } \\
\text { irrigation for knee osteoarthritis }\end{array}$ & $\begin{array}{l}\text { No differences between groups at } \\
1 \text { year; both improved on primary } \\
\text { outcome measures }\end{array}$ & Unknown & $\begin{array}{c}\text { Yes } \\
n=180\end{array}$ & 2 years \\
\hline $\begin{array}{l}\text { Buchbinder et } \\
\text { al. }(2009)^{10}\end{array}$ & $\begin{array}{l}\text { Vertebroplasty to sham surgery } \\
\text { for painful osteoporotic vertebral } \\
\text { fractures }\end{array}$ & $\begin{array}{l}\text { No differences between groups } \\
3 \text { months; both improved on primary } \\
\text { outcome measures }\end{array}$ & $36 \%$ & $\begin{array}{l}\text { No } \\
n=78 \\
\text { Target } \\
n=200\end{array}$ & 4.5 years \\
\hline $\begin{array}{l}\text { Firanescu et al. } \\
(2018)^{23}\end{array}$ & $\begin{array}{l}\text { Vertebroplasty to sham surgery } \\
\text { for acute osteoporotic vertebral } \\
\text { fractures }\end{array}$ & $\begin{array}{l}\text { No differences between groups during } \\
\text { 12-month follow-up; both improved on } \\
\text { primary outcome measures }\end{array}$ & $64 \%$ & $\begin{array}{l}\text { No } \\
\mathrm{n}=180 \\
\text { Target } \\
\mathrm{n}=200\end{array}$ & 2 years \\
\hline $\begin{array}{l}\text { Kallmes et al. } \\
(2009)^{28}\end{array}$ & $\begin{array}{l}\text { Vertebroplasty to sham surgery } \\
\text { for painful osteoporotic vertebral } \\
\text { compression fractures }\end{array}$ & $\begin{array}{l}\text { No differences between groups at } \\
1 \text { month; both improved on primary } \\
\text { outcome measures }\end{array}$ & $30 \%$ & $\begin{array}{l}\text { No } \\
n=131 \\
\text { Target } \\
n=294\end{array}$ & 4 years \\
\hline $\begin{array}{l}\text { Kroslak and } \\
\text { Murrell } \\
(2018)^{24}\end{array}$ & $\begin{array}{l}\text { Surgical excision of the } \\
\text { degenerative portion of the } \\
\text { extensor radialis brevis to sham } \\
\text { surgery for tennis elbow }\end{array}$ & $\begin{array}{l}\text { No differences between groups at } \\
6 \text { months; both improved on primary } \\
\text { outcome measures }\end{array}$ & Unknown & $\begin{array}{l}\text { No } \\
n=26 \\
\text { Target } \\
n=80\end{array}$ & 6 years \\
\hline $\begin{array}{l}\text { Moseley et al. } \\
(2002)^{8}\end{array}$ & $\begin{array}{l}\text { Arthroscopic debridement and } \\
\text { arthroscopic lavage to sham } \\
\text { surgery for knee osteoarthritis }\end{array}$ & $\begin{array}{l}\text { Neither interventional group was } \\
\text { superior to the sham surgery at } 2 \text { years }\end{array}$ & $40 \%$ & $\begin{array}{c}\text { Yes } \\
n=180\end{array}$ & 3 years \\
\hline $\begin{array}{l}\text { Roos et al. } \\
(2018)^{25}\end{array}$ & $\begin{array}{l}\text { Arthroscopic partial } \\
\text { meniscectomy to sham surgery } \\
\text { for degenerative knee meniscus } \\
\text { tears in young patients }\end{array}$ & $\begin{array}{l}\text { A greater improvement in the } \\
\text { intervention arm compared to sham } \\
\text { arm; the between-group difference was } \\
\text { not clinically significant }\end{array}$ & $43 \%$ & $\begin{array}{l}\text { No } \\
n=44 \\
\text { Target } \\
n=100\end{array}$ & 7 years \\
\hline $\begin{array}{l}\text { Schrøder et al. } \\
(2017)^{11}\end{array}$ & $\begin{array}{l}\text { Labral repair and biceps } \\
\text { tenodesis to sham surgery for } \\
\text { shoulder SLAP lesions }\end{array}$ & $\begin{array}{l}\text { No differences between groups at } \\
6 \text { months or } 2 \text { years; all three arms } \\
\text { improved on outcome measures }\end{array}$ & Unknown & $\begin{array}{l}\text { Yes } \\
\mathrm{n}=118 \\
\text { Target } \\
\mathrm{n}=120\end{array}$ & 6 years \\
\hline $\begin{array}{l}\text { Sihvonen et al. } \\
(2013)^{7,30}\end{array}$ & $\begin{array}{l}\text { Arthroscopic partial } \\
\text { meniscectomy to sham surgery } \\
\text { for degenerative meniscal tears }\end{array}$ & $\begin{array}{l}\text { No differences between groups at } \\
1 \text { year or } 2 \text { years; both improved on all } \\
\text { outcome measures }\end{array}$ & $78 \%$ & $\begin{array}{c}\text { Yes } \\
n=146\end{array}$ & 6 years \\
\hline
\end{tabular}

exceeded this threshold through the introduction of unnecessary steps in the sham including entering the joint space and exposing participants to the risk of infection, ${ }^{27}$ with no justification from the authors as to why this was necessary. Five trials, $5,9,11,23,28$ included other unnecessary steps such as irrigation, injection of anaesthetic and aspiration of synovial fluid, suggesting that these sham controls were not 'inert' but had a possible therapeutic effect. ${ }^{29}$

Consistent with poor evidence of a valuable, clinically relevant question to be answered by a sham surgery trial and/or the introduction of unnecessary steps in the sham, in six trials the risk of a placebo control appeared unjustified by the valuable knowledge to be gained.

Only four of the trials explicitly explained how participants were informed of the potential that they could receive sham surgery. In the remaining trials it can only be assumed that the participants were made aware of this during the informed consent process.

Characteristics of the included trials are presented in Table III and described below. Further detail on the methods and findings of each trial can be found in Appendix 1 (at the end).

These trials have significantly impacted the practice landscape of orthopaedics. ${ }^{30}$ Clinical guidelines now recommend against the use of many of the procedures described above, suggesting that the billions of dollars spent on these procedures annually should be put to better use. ${ }^{31,32}$ While these studies indicate that sham surgery trials can be feasible and provide valid data on the efficacy of some minor orthopaedic procedures, they also highlight the challenges facing sham surgery trials in orthopaedics. In particular are the common challenges of recruiting participants within reasonable time frames.

Moseley et al. ${ }^{8}$ reported that only $40 \%$ of eligible patients agreed to participate. Sihvonen et al. ${ }^{7}$ reported that $78 \%$ of eligible patients agreed to participate; however, the trial took almost 6 years to recruit the target sample of 146 . Schrøder et al. ${ }^{11}$ also took 6 years to recruit their sample, significantly exceeding normal funding timelines of 3-4 years. While Firanescu et al. ${ }^{23}$ reported that $64 \%$ of eligible patients agreed to participate and it only took 2 years to recruit their sample, they adjusted their target sample size from 200 to 180 and broadened their inclusion criteria part way through citing 'difficulties with recruitment', without further justification. This arguably changes the pretext of the study and may be considered a violation of the intention to treat criteria.

Buchbinder et al. ${ }^{10}$ reported that only $36 \%$ of eligible candidates agreed to participate. The trial took 4.5 years to recruit a sample of 78 and this was 122 participants short of their target ${ }^{33}$ raising 
Table IV. Sham surgery trials with unpublished findings

\section{Study group}

Burke et al.

Registry i.d. ${ }^{1}$

NCT01799876

\section{Dragoo et al.}

Registry i.d.

NCT03014401

Ferreira et al.

Registry i.d. ${ }^{2}$

ACTRN126170-

$00884303 p$

Giori et al.

Registry i.d.

NCT01931735

Grisby et al.

Registry i.d.

NCT01129921

Hanvold et al.

Registry i.d.

NCT02636881

Matache et al.

Registry i.d.

NCT02236689

Paavola et al.

Registry i.d.

NCT00428870

Reischling et al.

Registry i.d.

NCT03112200

Risberg et al.

Registry i.d.

NCT02692807

Ryösä et al.

Registry i.d.

NCT02885714

VertiFlex

incorporated

Registry i.d

NCT02079038
Key dates

Comparison

Start date 2013 Autologous cell surgery (regenerative cells obtained from autologous fat are administered in the knee at microfracture site) to sham surgery for knee articular cartilage defect grade iii or iv

Start date 2016 Arthroscopic debridement with stem cell transplantation to sham surgery for mild-moderate knee osteoarthritis

Start date 2017 Decompressive spinal surgery via laminectomy or laminotomy to sham surgery for lumbar spinal stenosis

Start date 2013 Arthroscopic meniscectomy to Completion sham surgery for degenerative knee date 2017 meniscus tears

Start date 2010 Fluoroscopic percutaneous lumbar decompression to sham surgery for

Completion date 2013 moderate to severe lumbar central canal stenosis

Start date 2016 Autologous chondrocyte implantation to sham surgery for knee cartilage injury

Start date 2014 Arthroscopic tennis elbow release to sham surgery for tennis elbow

Start date 2005 Arthroscopic acromioplasty to sham Recruitment surgery for subacromial impingement completed syndrome 2017

Start date 2017

Fluoroscopically assisted subchondroplasty procedure to sham surgery for bone marrow oedema in the knee

Start date 2016 Arthroscopic surgical procedures to sham surgery for patients with femoroacetabular impingement and/ or labral tears

Start date 2016 Rotator cuff repair to sham surgery and supervised specific exercises for acute rotator cuff tear related to trauma

Start date 2014 Totalis $^{\mathrm{TM}}$ direct decompression procedure to sham surgery for lumbar spinal stenosis

\section{Outcome/status as of June 2018}

Target sample size

Completed data collection. Main findings paper yet to be published

48

Recruiting

Seeking ethics approval

This trial was funded as a sham-surgery trial. However following publication of like trials, it was deemed unethical to continue and the trial converted to an observational study

Completed data collection. Main findings paper yet to be published

Recruiting

Completed data collection. Protocol

paper published 2017. Main findings paper yet to be published

Recruiting

Recruiting

Recruiting

Completed data collection. Main findings paper yet to be published
Completion date 2017

${ }^{1}$ Registry i.d. is the trial number as registered on the website clinicaltrials.gov

${ }^{2}$ Registry i.d. is the trial number as registered on the ANZCTR.org.au website

concern as to whether the study was adequately powered to support its conclusions. Similarly, Kallmes et al. ${ }^{28}$ reported that only $30 \%$ of eligible candidates agreed to participate. Citing initial difficulties with recruitment, the sample size was reduced from 294 to 131. The length of follow-up was also shortened from 2 years to 1 year; however, the 1 -year findings have still not been published, nine years later. To date, only the 3-month follow-up outcomes have been reported and this lack of long-term follow-up can be considered a key limitation, particularly given that many surgical procedures do not reach their peak effectiveness until 6-12 months. ${ }^{34}$ The Kallmes et al. trial also seems to have suffered from a high rate of non-compliance to treatment, with suboptimal blinding procedures. At 2 weeks, 63\% of those in the sham arm correctly guessed their allocation compared to $51 \%$ in the intervention arm. Further, $74 \%$ of the 27 participants who elected to cross over from the sham correctly guessed their allocation compared to $55 \%$ of those who did not cross over.

The Beard et al. ${ }^{6}$ trial also suffered from a high rate of noncompliance to treatment. Only $58 \%$ of the people randomised to the sham arm were treated as per protocol due to withdrawal from the study, immediate crossover to the intervention arm or not yet having received the sham intervention by the primary end 
point at 6 months. ${ }^{6}$ Indeed at 6 months, $40 \%$ of participants in the sham arm had not yet undergone the intervention (were awaiting surgery), four times as many as in the intervention arm. Given that follow-up was planned for 6 months post-randomisation rather than post-intervention, this significantly confounds interpretation of the trial results. Beard et al. planned to embed a qualitative investigation into their trial to identify sources of recruitment difficulties and suggest changes to improve informed consent and randomisation. However, the qualitative findings have not been reported. The trial also suffered from difficulties with recruitment, doubling the number of centres they recruited from to achieve their target sample size within 3 years. ${ }^{35}$

Roos et al. received funding in 2010 to compare arthroscopic partial meniscectomy to sham surgery for degenerative meniscus tears. In 2013 they published a protocol paper ${ }^{36}$ and in 2014 they published a paper describing their experiences with recruitment. ${ }^{16}$ In all, $43 \%$ of eligible candidates agreed to participate. The reasons for non-participation were: not wanting sham surgery; the risk of undergoing a secondary operation if allocated to the sham surgery; and not wanting surgery at all. Among the 40 individuals recruited, the most common reason for participating was 'contribution to research'. However, the trial fell short of its target sample size $(n=100)$ and in 2017 the trial was terminated in the ClinicalTrials. gov registry due to 'insufficient recruitment'. A paper published in 2018 reported the findings from 44 patients enrolled in the study. ${ }^{25}$ Ten of the 22 patients randomised to the sham arm were non-blinded and of these, eight chose to cross over to receive the intervention, further confounding interpretation of the trial results.

\section{Findings from trials without a published main findings paper}

We identified a further 12 sham surgery trials for minor orthopaedic procedures that are currently underway, have been recently completed with the findings yet to be published, or that have been abandoned (Table IV).

As with the published trials, similar difficulties with recruitment can be seen among these trials. Paavola et al. compared arthroscopic acromioplasty to sham surgery for subacromial impingement syndrome and took eight years to recruit the target sample size of 210 participants. Notably a protocol paper was not published until 2017,37 four years after the completion of data collection, and the main findings are yet to be reported. Grisby et al. compared fluoroscopic percutaneous lumbar decompression to sham surgery for moderate to severe lumbar central canal stenosis and are also yet to publish their main findings in a peerreviewed journal, eight years after commencing their trial. Despite the small size $(n=40)$, documentation on the clinicaltrials.gov registry reports difficulties with patient retention in the sham arm. Matache et al. registered a trial in 2014 comparing arthroscopic tennis elbow release to sham surgery for tennis elbow with a projected completion date in 2017. A protocol paper was published in 2016,38 recruitment was only commenced the same year, and the projected completion date has been extended by four years according to the clinicaltrials.gov website. The trial by Ferreira et al. highlights potential difficulties with obtaining ethical approval for a sham surgery trial. The trial was registered in May 2017 and the first participant was expected to be enrolled by September 2017. In May 2018, the trial has still not received ethical approval.

\section{Discussion}

Recruiting participants into orthopaedic trials is a well-known challenge. Two interventions that have different benefit-to-harm profiles can result in strong preferences for one intervention, leading both patients and surgeons to decline participation in a trial. ${ }^{39}$ Adding a sham component to the trial further complicates recruitment. Among patients, willingness to participate in a clinical trial is associated with a higher education level. ${ }^{40}$ The selective non-participation of people with less education reduces the generalisability of trial findings; thus an extra effort may be required to explain the rationale for a sham surgery trial to people with lower health literacy. Among surgeons, willingness to recruit patients for a sham surgery trial may depend on design preferences. Survey responses from 189 members of the British Orthopaedic Trainees Association revealed that of those who preferred a sham-control design over an active-control, 30\% stated they would 'definitely' recruit patients for a sham surgery trial, and $56 \%$ stated they would 'probably'. ${ }^{41}$ Surgeon willingness to participate in a clinical trial may also depend, at least in part, on remuneration. A pragmatic obstacle for sham surgical trials in orthopaedics is whether payers will reimburse for it. While insurance payers commonly cover the cost of treatment in research studies for more acceptable procedures, the cost of sham surgery often must come from research funds. ${ }^{42}$ The costs associated with extra recruitment efforts, extended recruitment timelines and reimbursement for surgeons present a significant challenge for sham surgery trials in orthopaedics.

A recent investigation into clinical trials in Australia found that $50 \%$ of current research investment by the Australian National Health and Medical Research Council is spent on studies that do not publish a protocol paper and/or main results paper during the funding period. ${ }^{43}$ Researchers are obliged to carefully consider the feasibility of conducting a sham surgery trial in a major orthopaedic procedure, before drawing on limited research funds. Based on the findings of this review, we recommend that prior to conducting a sham surgery trial, it is important to determine the conditions under which surgeons and patients would find participation in a sham surgery trial for a major orthopaedic procedure acceptable.

Insight into the complex decision-making processes involved in decisions to participate, in addition to preferred trial designs, can be provided by exploratory methodologies such as qualitative interviews and discrete choice experiments. ${ }^{44,45}$ For example,

1. Discrete choice experiments
\begin{tabular}{|l|l|l|}
\hline Procedure & Remuneration & Trial design \\
\hline Choice A & Choice $A^{\prime}$ & Choice A" \\
\hline Choice B & Choice B' & Choice B" \\
\hline Choice C & Choice C' & Choice C" \\
\hline
\end{tabular}

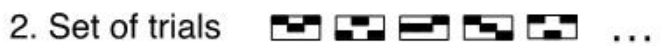

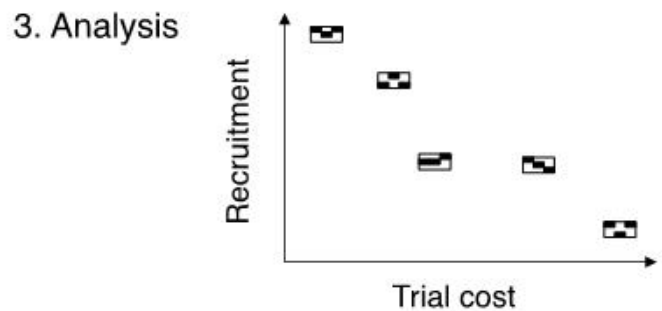

Figure 4. Example study where discrete choice experiments generate surgeon preferences for variables such as type of procedure, amount of remuneration and trial design e.g. cross over, parallel design (1). These choice experiments are then trialled (2) and analysed for correlations between for example, trial cost and surgeon recruitment rates (3) to determine the optimal trial designs. 
systematically structured trade-off questions can provide information about patient and surgeon willingness to accept tradeoffs among features of specific trials with different characteristics. ${ }^{46}$ Are surgeons more willing to participate in a sham surgery trial if the trial involves unicompartmental as opposed to total joint replacement? If the trial involves a crossover rather than parallel design? If they are remunerated for performing a sham procedure at the same rate as an active procedure? By evaluating series of pairs of hypothetical options, patterns of choices can reveal the underlying preferences associated with choosing to participate in a sham surgery trial. Based on these preferences, health economic modelling can simulate the costs of conducting a sham surgery trial including estimated cost functions of recruitment and estimates of improvement in recruitment rates. To continue the example above, if a certain trial design is preferred by surgeons, this may produce higher rates of recruitment, and the change in cost can be quantified via simulation models (Figure 4).

\section{Conclusion}

By searching the published and unpublished literature, we have presented a comprehensive picture of the landscape of sham surgery trials in orthopaedics. We captured the full range of sham surgery trials with published main findings by using broad terms and having two reviewers screen titles and abstracts. Search engines differed between clinical trial registries, preventing an exhaustive search of the unpublished literature.

Only one of the ten included trials with a published main findings paper was free from risk of bias; all others were at risk of bias. The findings illustrate that effective randomisation, concealment and blinding procedures are possible in orthopaedic sham surgery trials. However, the incidence of crossover from the sham arm was high among the included studies suggesting that blinding was not always effective. We recommend that all future trials assess and report the effectiveness of blinding procedures by asking participants which intervention they believed they received and why. The findings particularly highlight the challenge of participant recruitment in sham surgery trials. Low recruitment is not only a source of bias and threat to external validity, it also places trials at risk of abandonment. Closing a trial due to low recruitment rates prior to generating valuable knowledge and after exposing patients to the risks of sham surgery presents a significant ethical concern.

Findings from the ethical assessment suggest that the benefits of a sham control were outweighed by the risks in most of the included trials with a published main findings paper. Only two of the ten trials fulfilled all six criteria of the ethical assessment. Given that the trials had all received ethics approval, this raises questions about the criteria ethics committees employ when assessing risk in sham surgery trials. In seven trials, the sham control exposed participants to risk beyond accepted thresholds, with no justification from the authors as to why this was necessary. Future trials should limit the sham control to anaesthetic and skin incision, not only to prevent exposing participants to unnecessary risks, but to also ensure that the findings can yield meaningful knowledge about treatment efficacy.

All of the included trials involved minor orthopaedic procedures; the challenges identified in this review will be amplified for major orthopaedic procedure. Feasibility studies based on patient and surgeon preferences may assist in the development of minimum criteria for funders, assessors and ethics boards to determine whether to support the conduct of future sham surgery trials in major orthopaedic procedures.

\section{Acknowledgements}

Michelle Dowsey is supported by a Career Development Fellowship from the National Health and Medical Research Council of Australia. Peter Choong and Michelle Dowsey hold a National Health and Medical Research Council Centre for Research Excellence Grant in Total Joint Replacement (1116325).

\section{Ethics statement}

The authors declare that this submission is in accordance with the principles laid down by the Responsible Research Publication Position Statements as developed at the 2 nd World Conference on Research Integrity in Singapore, 2010. This article did not involve human or animal subjects.

\section{Appendix 1. Description of sham surgery trials with published main findings}

Beard et al. ${ }^{6}$ compared arthroscopic subacromial decompression to sham surgery and no treatment in 313 people who had experienced subacromial shoulder pain for $>3$ months duration; had undertaken an exercise intervention and at least one steroid injection; and had intact rotator cuff tendons. Arthroscopic decompression was performed under general anaesthetic and involved the removal of the bursa and soft tissue in the subacromial space; release of the coracoacromial ligament; and removal of the subacromial bone spur. Sham surgery involved arthroscopy, performed under general anaesthetic. The procedure involved inspection and irrigation of the glenohumeral joint and subacromial bursa. 'No treatment' involved monitoring patients at 3 months after entering the study with a specialist shoulder clinician but no other prescribed treatment. The success of patient blinding was not reported. Outcome assessments were performed by a blind assessor. No differences in pain, function, range of motion or quality of life were found between the decompression and sham group, with both showing improvements on most measures compared to the no treatment group at 6 months and 1 year. There were six study-related complications (frozen shoulders), two in each group. A further two participants in the sham group required further surgery for pain; one underwent decompression and the other a superior labrum anterior posterior debridement.

Bradley et $\boldsymbol{a l} .{ }^{9}$ compared needle tidal irrigation to sham irrigation in 180 people who had experienced knee pain for $\geq 1$ year and met the American College of Rheumatology clinical or clinical plus radiographic criteria for knee osteoarthritis. Tidal irrigation involved the insertion of a needle into the joint capsule, the aspiration of bupivacaine-containing fluid and injection of fresh saline into the knee that was withdrawn and ejected repeatedly. Sham irrigation involved the insertion of a needle towards but not through the joint capsule, and the injection and ejection of saline in the subcutaneous tissue. Patients received topical anaesthesia, and a vertical drape was used to obscure their view of the procedure. Blinding of patients was successful, with almost $90 \%$ of participants in the tidal irrigation and sham irrigation groups believing they had received tidal irrigation. Outcome assessments were conducted by a nurse blinded to patients' allocation. No differences in WOMAC score pain and function were found between groups at the 3-, 6- or 12-month follow-up. No significant adverse events were reported. One patient reported increased knee pain at 6 months.

Buchbinder et $\mathbf{a l} .^{10}$ compared vertebroplasty to sham surgery in 78 people who had experienced back pain for $<12$ months duration and had one or two recent vertebral fractures of grade 1 or higher according to Genant grading system, with evidence of oedema, a fracture line, or both on MRI. Percutaneous vertebroplasty involved an incision into the skin over the posterior lamina; the insertion of a 13-gauge needle into the fractured vertebral body; and the injection of polymethylmethacrylate (PMMA) into the vertebral body by a radiologist. Sham surgery involved the same skin incision and the insertion of a 13-gauge needle to rest on the lamina. The vertebral body was gently tapped to simulate vertebroplasty and the PMMA was prepared to permeate the smell through the room. The success of patient blinding was not reported. Outcome assessments were performed by a blind assessor. No differences in pain, function or quality of life were found between the two groups at the 3 -month end point. 
Three patients who received vertebroplasty and four who received sham surgery reported an incident clinical vertebral fracture 6 months postsurgery. One patient who received vertebroplasty and two who received sham surgery reported new rib fractures at one week post-surgery. One patient who received vertebroplasty developed a new fracture and osteomyelitis necessitating surgical drainage and antibiotic treatment two weeks post-surgery.

Firanescu et al. ${ }^{23}$ compared vertebroplasty to sham surgery in 180 people with 1-3 acute osteoporotic vertebral compression fractures of $<9$ weeks duration, a pain score of $>5 / 10$ on the VAS, diminished bone density and $15 \%$ or more loss of vertebral height and bone oedema on MRI. All participants underwent local infiltration with $1 \%$ lidocaine into each pedicle and $0.25 \%$ bupivacaine prior to randomisation. Vertebroplasty involved incisions at the level of the vertebral body and bone biopsy needles position bilaterally using standard transpedicular placements. A cement injector was attached to the needles and PMMA was injected until cement leakage was noticed on CT. Sham surgery involved the same skin incisions and placement of bone biopsy needles only, plus simulation of the PMMA odour. Participants received local anaesthetic; 20 received conscious sedation. Blinding was successful with $81 \%$ of patients in the sham arm and $82 \%$ in the intervention arm believing they had undergone vertebroplasty. Outcome assessments were performed by an assessor blinded to patients' allocation. No differences in pain, function or quality of life were found between groups at any follow-up point up to 12 months. Two adverse events were reported in the vertebroplasty group. One patient developed respiratory insufficiency one day post-intervention, related to underlying pulmonary disease. Another patient had a vasovagal reaction during the procedure that resolved spontaneously.

Kallmes et $\boldsymbol{a l} .^{28}$ compared vertebroplasty to sham surgery in 131 people with back pain of $\geq 3$ on a scale of 0 to 10 , who had not responded to standard medical therapy and had confirmed osteoporosis or osteopaenia and one or two vertebral fractures in vertebrae T4 to L5 of less than 1 year old. All patients underwent infiltration of the skin and subcutaneous tissues over the pedicle of the target vertebra(e) with $1 \%$ lidocaine and infiltration of the periosteum of the pedicles with $0.25 \%$ bupivacaine prior to randomisation. Percutaneous vertebroplasty involved passing an 11- or 13-gauge needle into the central aspect of the target vertebra and the infusion of PMMA into the vertebral body under lateral fluoroscopy. Sham surgery involved pressure on the patient back and simulation of the PMMA odour. Patients in both arms received conscious sedation. At 2 weeks, $51 \%$ of the vertebroplasty arm and $63 \%$ of patients in the sham arm correctly guessed their allocation. Twenty of 27 patients who crossed over from the sham to the intervention correctly guessed their allocation. Outcome assessments were performed by a blind assessor. No differences in disability, pain or quality of life were found between the two groups at the 1-month end point. One patient in the vertebroplasty arm received an injury to the thecal sac during the procedure and was hospitalised.

Kroslak and Murrel ${ }^{24}$ compared surgical excision of the degenerative portion of the extensor radialis brevis to sham surgery in 26 patients with a clinical diagnosis of lateral epicondylitis that had persisted for $>6$ months despite conservative management. The surgical procedure involved the Nirschl mini-open technique without cortical drilling. A 2.5 lateral incision was made over the lateral epicondyle. The pathological tendinosis tissue of the extensor carpi radialis brevis (ECRB) was excised. Sham surgery involved the same skin incision and scalpel dissection and traction of tissue to enable visualisation of the ECRB tendon before proceeding to closure. Patients in both groups received local infiltration and sedation. The effectiveness of blinding was not reported. Outcome assessments were performed by an assessor blinded to patients' allocation. No difference in pain or function were found between groups at 6 months. No adverse events were reported.

Moseley et al. ${ }^{8}$ compared arthroscopic debridement and arthroscopic lavage to sham surgery in 180 patients who had experienced knee pain for $\geq 6$ months and met the American College of Rheumatology criteria for knee osteoarthritis. Arthroscopic debridement involved a diagnostic arthroscopy followed by joint lavage with $10 \mathrm{~L}$ of fluid, shaving of the rough articular cartilage, removal of loose debris, trimming of torn or degenerated meniscal fragments and smoothing of remaining meniscus to a firm, stable rim. Bone spurs blocking full extension were shaved smooth. Arthroscopic lavage involved a diagnostic arthroscopy followed by joint lavage with $10 \mathrm{~L}$ of fluid. Debris that could be flushed out through the arthroscopic cannulas was removed. Sham surgery involved a partial anaesthesia and three $1 \mathrm{~cm}$ incisions made in the skin. The surgeon asked for instruments and manipulated the knee as if an arthroscopy was being conducted. No instrument entered the incisions. Blinding of patients was successful with $13 \%$ of participants in each of the three groups believing they had undergone the placebo procedure. Outcomes assessments were performed by an assessor blinded to patients' allocation. No difference in pain or function were found between any of the three groups up to 2 years later. No significant adverse events were reported.

Roos et al. ${ }^{25}$ compared arthroscopic partial meniscectomy to sham surgery in 44 people aged $35-55$ years with knee pain for $>2$ months and a medial meniscus lesion confirmed on MRI. Arthroscopic partial meniscectomy was performed under general anaesthesia combined with local anaesthesia. Standard procedures were followed, and the arthroscope was inserted with the aim of preserving as much tissue as possible. The sham group received the same anaesthesia and the knee was manipulated as if a real arthroscopy was performed. No instruments entered into the incisions. Sixteen participants (36\%) were non-blinded prior to the 2-year follow-up, by either the study nurse, the participants' GP, or the treating surgeon due to suspicion of an adverse event or persistent pain. Outcome assessments were performed by a blind assessor. At 3 months, both groups had clinically important improvements in pain and function with no significant difference between groups. Improvements were sustained at the 2-year follow-up. While greater improvements were documented in the partial meniscectomy group at 2 years, the between group difference was not clinically relevant. Four knee-related adverse events were recorded, all in the surgery group, which resulted in two re-arthroscopies, one partial meniscectomy and one anterior cruciate ligament reconstruction.

Schrøder et al. ${ }^{11}$ compared labral repair and biceps tenodesis to sham surgery in 118 people who had experienced shoulder pain for $>3$ months; were unresponsive to conservative treatment and had clinical findings and MRI results indicating type II SLAP lesions. An arthroscopic shoulder examination was performed on all patients under a general anaesthetic. The labral repair involved debridement of the superior glenoid rim with a motorised shaver followed by percutaneous placement of a drill guide and anchors through the supraspinatus myotendinous junction, placed posterior to the biceps root. The biceps tenodesis involved a tenotomy at the biceps insertion performed under arthroscopic vision with a spinal needle and with $a<2 \mathrm{~cm}$ skin incision to mimic the other groups. Sham surgery involved a diagnostic shoulder arthroscopy. All three groups received exercise rehabilitation. Only $73 \%$ of patients who received sham surgery believed they were repaired, compared to $89 \%$ of patients who received labral repair, and $97 \%$ who received biceps tenodesis, suggesting that blinding was not completely achieved. Outcome assessments were performed by a single blind assessor. No differences in pain, function or quality of life were found between the three groups, with all showing significant improvement at 6 and 24 months. No serious adverse events were reported; ten patients reported ongoing stiffness (capsulitis) five in the labral repair group, four in the biceps tenodesis group and one in the sham surgery group.

Sihvonen et al..$^{7,30}$ compared arthroscopic partial meniscectomy with sham surgery in 146 patients who had experienced knee pain for $>3$ months; were unresponsive to conservative treatment; and had clinical findings consistent with a degenerative tear of the medial meniscus without knee osteoarthritis as defined by the American College of Rheumatology or Kellgren-Lawrence grade $>1$. All patients received a diagnostic arthroscopy of the knee to confirm eligibility prior to randomisation. Arthroscopic partial meniscectomy involved the removal of damaged and loose parts of the meniscus until solid meniscal tissue was reached using a mechanised shaver and meniscal punches. Sham surgery involved mimicking the sounds and sensations of the arthroscopic partial meniscectomy by the surgeon asking for all instruments and manipulating the knee as if it was performing the true surgery. The surgeon pushed a mechanised shaver, minus the blade, against the patella on the outside of the knee and used suction. Blinding was successful with no significant difference between groups when asked which intervention they believed they had received. Outcome assessments were performed by an assessor blinded to patients' allocation. No differences in pain or function were found between groups at 12 months or 2 years, with both groups improving in all outcome measures. One patient who received partial meniscectomy experienced an infection at four months.

\section{References}

1. Sackett D, Rosenberg W, Gray J, et al. Evidence based medicine: What it is and what it isn't. BMJ. 1996;312:71-72.https://doi. org/10.1136/bmj.312.7023.71

2. Lim H, Adie S, Naylor J, et al. Randomised trial support for orthopaedic surgical procedures. PLoS One. 2014;9(6):e96745. https://doi.org/10.1371/journal.pone.0096745

3. Lin C, Day R, Harris I, et al. Comparative efficacy trials with no placebo group cannot determine efficacy. BMJ. 2015;350(h3292).https://doi.org/10.1136/bmj.h3292 
4. Krogsboll L, Hrobjartsson A, Gotzsche P. Spontaneous improvement in randomised clinical trials: meta-analysis of three-armed trials comparing no treatment, placebo and active intervention. BMC Med Res Methodol. 2009;9(1).https://doi. org/10.1186/1471-2288-9-1

5. Enck $P$, Bingel $U$, Schedulowski $M$, et al. The placebo response in medicine: minimize, maximize or personalize. Nat Rev 2013;12:191-2014.https://doi.org/10.1038/nrd3923

6. Beard D, Rees J, Cook J, et al. Arthroscopic subacromial decompression for subacromial shoulder pain (CSAW): a multicentre, pragmatic, parallel group, placebo-controlled, three group, randomised surgical trial. Lancet. 2018;391:329-228. https://doi.org/10.1016/S0140-6736(17)32457-1

7. Sihvonen R, Paavola M, Malmivaara A, et al. Arthroscopic partial meniscectomy versus sham surgery for a degenerative meniscal tear. The N Engl J Med. 2013;396(26).https://doi.org/10.1056/ NEJMoa1305189

8. Moseley B, O'Malley K, Petersen J. A controlled trial of arthroscopic surgery for osteoarthritis of the knee. The $N$ Eng J Med. 2002;347:81-88.https://doi.org/10.1056/NEJMoa013259

9. Bradley J, Heilman D, Katz B. Tidal irrigation as treatment for knee osteoarthritis: A sham-controlled, randomized, doubleblinded evaluation. Arthritis Rheum. 2002;46:100-108.10.1002/ art.10037

10. Buchbinder $R$, Osborne $R$, Ebeling $P$, et al. A randomized trial of vertebroplasty for painful osteoporotic vertebral fractures. The $N$ Engl J Med. 2009;361(6):557-68.https://doi.org/10.1056/ NEJMoa0900429

11. Schrøder CP, Skare O, Reikeras $O$, et al. Sham surgery versus labral repair or biceps tenodesis for type II SLAP lesions of the shoulder: a three-armed randomised clinical trial. $\mathrm{Br} J$ Sports Med. 2017;51(24):1759-66.https://doi.org/10.1136/ bjsports-2016-097098

12. Fischgrund JS, Rhyne A, Franke J, et al. Intraosseous basivertebral nerve ablation for the treatment of chronic low back pain a prospective randomized double-blind sham-controlled multi-center study. Eur Spine J. 2018.https://doi.org/10.1007/ s00586-018-5496-1

13. Wartolowska K, Beard D, Carr A. Attitudes and beliefs about placebo surgery among orthopaedic shoulder surgeons in the United Kingdom. PLoS One. 2014;9(3):e91699.https://doi. org/10.1371/journal.pone.0091699

14. Wartolowska K, Judge A, Hopewell $S$, et al. Use of placebo controls in the evaluation of surgery: systematic review. BMJ. 2014;348(g3253).https://doi.org/10.1136/bmj.g3253

15. Dowrick $A$, Bhandari $M$. Ethical issues in the design of randomised trials: To sham or not to sham. J Bone Joint Surg Am. 2012;94(Suppl 1):7-10.https://doi.org/10.2106/JBJS.L.00298

16. Hare $K$, Lohmander $L$, Roos $E$. The challenge of recruiting patients into a placebo-controlled surgical trial. Trials. 2014;15(167). https://doi.org/10.1186/1745-6215-15-167

17. World Medical Association. World Medical Association Declaration of Helsinki ethical principles for medical research involving human subjects. The Journal of the AMA. 2013;310(20):2191-914

18. Tenery R, Rakatansky H, Riddick F, et al. Surgical 'placebo' controls. Ann Surg. 2002;235(2):303-307

19. Moher D, Liberati A, Tetzlaff J, et al. Preferred Reporting Items for Systematic Reviews and Meta-Analyses: The PRISMA Statement. PLoS Med. 2009;6(7):e1000097.https://doi. org/10.7326/0003-4819-151-4-200908180-00135

20. Higgins JP, Altman DG, Gotzsche PC, et al. The Cochrane Collaboration's tool for assessing risk of bias in randomised trials. BMJ. 2011;343:d5928.https://doi.org/0.1136/bmj.d5928

21. Horng S, Miller FG. Ethical framework for the use of sham procedures in clinical trials. Crit Care Med. 2003;31(3 Suppl):S126-130.https://doi.org/10.1097/01. CCM.0000054906.49187.67

22. Probst P, Grummich K, Harnoss JC, et al. Placebo-controlled trials in surgery: a systematic review and meta-analysis Medicine (Baltimore). 2016;95(17):e3516.10.1097/ MD.0000000000003516

23. Firanescu CE, de Vries J, Lodder $\mathrm{P}$, et al. Vertebroplasty versus sham procedure for painful acute osteoporotic vertebral compression fractures (VERTOS IV): randomised sham controlled clinical trial. BMJ. 2018;361:k1551.https://doi.org/10.1136/bmj. k1551

24. Kroslak M, Murrell G. Surgical treatment of lateral epicondylitis The Am J Sports Med. 2018;46(5):1106-1113.https://doi. org/10.1177/0363546517753385

25. Roos E, Hare K, Nielsen $S$, et al. Better outcome from arthroscopic partial meniscectomy than skin incisions only? A sham-controlled randomised trial in patients aged 35-55 years with knee pain and an MRI-verfied meniscal tear.
BMJ Open. 2018;8(e019461).http://dx.doi.org/10.1136/ bmjopen-2017-019461

26. Horng S, Miller F. Is placebo surgery unethical? The N Engl J Med. 2002;347:137-39. 10.1056/NEJMsb021025

27. Balato $G$, Di Donato $\mathrm{SL}$, Ascione $\mathrm{T}$ et al Knee septic arthritis after arthroscopy: incidence, risk factors, functional outcome, and infection eradication rate. Joints. 2017;5(2):107-13.10.1055/s-0037-1603901

28. Kallmes D, Comstock B, Heagerty $P$, et al. A randomized trial of vertebroplasty for osteoporotic spinal fractures. The N Engl J Med. 2009;36(16):569-79.10.1056/NEJMoa0900563

29. Courtney $P$, Doherty $M$. Joint aspiration and injection and synovial fluid analysis. Best Pract Res Clin Rheumatol. 2013;27(2):137-69.10.1016/j.berh.2013.02.005

30. Amin N, Hussain W, Ryan J, et al. Changes within clinical practice after a randomized controlled trial of knee arthroscopy for osteoarthritis. The Orthop J Sports Med. 2017;5(4).https://doi. org/10.1177/2325967117698439

31. Siemieniuk R, Harris I, Agoritsas T, et al. Arthroscopic surgery for degenerative knee arthritis and meniscal tears: a clinical practice guideline. BMJ. 2017;357(1982).10.1136/bmj.j1982

32. National Institute for Health and Care Excellence. Osteoarthritis: care and management in adults. In. London: NICE; 2018.

33. Buchbinder R, Osborne R, Ebeling $P$, et al. Efficacy and safety of vertebroplasty for treatment of painful osteoporotic vertebral fractures: a randomised controlled trial. BMC Musculoskelet Disord. 2008:9(156).10.1186/1471-2474-9-156

34. Kennedy D, Stratford P, Riddle D, et al. Assessing recovery and establishing prognosis following total knee arthroplasty. Phys Ther 2008;88(1):22-32.10.2522/ptj.20070051

35. Beard D, Rees J, Rombach I, et al. The CSAW Study (Can Shoulder Arthroscopy Work?) - a placebo-controlled surgical intervention trial assessing the clinical and cost effectiveness of arthroscopic subacromial decompression for shoulder pain: study protocol for a randomised controlled trial. Trials. 2015;16(210).10.1186/ s13063-015-0725-y

36. Hare K, Lohmander S, Christensen R, et al. Arthroscopic partial meniscectomy in middle-aged patients with mild or no knee osteoarthritis: a protocol for a double-blind randomized sham-controlled multi-centre trial. BMC Musculoskel Disord. 2013;14(71)

37. Paavola M, Malmivaara A, Taimela S, et al. Finnish Subacromial Impingement Arthroscopy Controlled Trial (FIMPACT): a protocol for a randomised trial comparing arthroscopic subacromial decompression and diagnostic arthroscopy (placebo control), with an exercise therapy control, in the treatment of shoulder impingement syndrome. BMJ Open. 2017;7(5):e014087.10.1136/ bmjopen-2016-014087

38. Matache BA, Berdusco R, Momoli F, et al. A randomized, doubleblind sham controlled trial on the efficacy of arthroscopic tennis elbow release for the management of chronic lateral epicondylitis. BMC Musculoskelet Disord. 2016;17(1) (no pagination)(364).10.1186/s12891-016-1093-9

39. Ergina $P$, Cook J, Blazeby J, et al. Challenges in evaluating surgical innovation. Lancet. 2009;26(374):1097-104.10.1016 S0140-6736(09)61086-2

40. Kerman HM, Deshpande BR, Selzer F, et al. Willingness of older adults to participate in a randomized trial of conservative therapies for knee pain: A prospective preference assessment. Contemp Clin Trials Commun. 2018;9:93-97.10.1016/j. conctc.2017.12.006

41. Baldwin M, Wartolowska K, Carr A. A survey on beliefs and attitudes of trainee surgeons towards placebo. BMC Surgery. 2016;16(27).10.1186/s12893-016-0142-5

42. Katz J, Losina $E$, Lohmander L. OARSI clinical trial recommendations: Design and conduct of clinical trials of surgical interventions for osteoarthritis. Osteoarthr Cartil. 2015;23:798-802.10.1016/j.joca.2015.02.024

43. Strand L, Clarke P, Graves N, et al. Time to publication for publicly funded clinical trials in Australia: an observational study. BMJ Open. 2017;7(e012212).http://dx.doi.org/10.1136/ bmjopen-2016-012212

44. Augustovski F, Beratarrechea A, Irazola V, et al. Patient preference for biologic agents in rheumatoid arthritis: A discrete choice experiment. Value Health. 2013;16(2):385-393.10.1016/j. jval.2012.11.007

45. Campbell M, Entwistle V, Cuthbertson B, et al. Developing a placebo-controlled trial in surgery: Issues of design, acceptability and feasibility. Trials. 2011;12(50).10.1186/1745-6215-12-50

46. Lancsar E, Louviere J. Conducting discrete choice experiments to inform healthcare decision making: a user's guide. Pharmacoeconomics. 2008;26:661-77.https://doi. org/10.2165/00019053-200826080-00004 